\title{
Editorial
}

Chirurg 2014 · 85:1097

DOI 10.1007/s00104-014-2897-5

Online publiziert: 16. Oktober 2014

๑) Springer-Verlag Berlin Heidelberg 2014
H. Dralle

Universitätsklinik für Allgemein-, Viszeral- und Gefäßchirurgie,

Universitätsklinikum Halle, Medizinische Fakultät, Halle (Saale)

\section{Komplikationen in der Chirurgie}

\section{Jahresthema 2015 in Der Chirurg}

Technische Fortschritte und krankheitsorientierte schonende Chirurgie haben die Heilungschancen in vielen Bereichen der Chirurgie erheblich verbessert und die eingriffsbezogenen Belastungen des Patienten verringert. Sie lösen nicht selten Anerkennung und sogar Bewunderung aus. Schon immer aber waren auch die unerwünschten Operationsfolgen Bestandteil der Chirurgie, die Demut erzeugende [1] andere Seite der ansonsten vielfach glänzenden Medizinmedaille, gleichsam das Yin und Yang des Chirurgen.

Doch daß mich die besten Erfolge meiner Kunst befriedigten, kann ich nicht sagen. Was ich nicht kann, was mir mißglückt, das quält und wurmt mich. ([2], S. 152-153)

...schrieb kein Geringerer als der sicher nicht wenig Erfolg gewöhnte Theodor Billroth 43-jährig am 25.10.1872 an den Anatomieprofessor Wilhelm His in Leipzig.

Nicht jede negative Operationsfolge ist eine vermeidbare Komplikation. Doch gerade die Fortschritte der Medizin, be sonders auch der Chirurgie, haben wahrscheinlich dazu beigetragen, dass immer mehr Menschen unerwünschte Operationsfolgen als vermeidbare Komplikation verstehen und rechtliche Klärung anstreben. Der Krankenhausreport 2014 schätzt, dass etwa die Hälfte der unerwünschten Ereignisse im Krankenhaus (2-4\% von 5-10\%) vermeidbar seien, und kalkuliert, dass auf 1000 Krankenhauspatienten ein Todesfall durch einen Behandlungsfehler käme. Die Zahl der Todesfälle durch Fehler im Krankenhaus sei damit rund 5-mal so hoch wie die der Verkehrstoten [3]. Der
Chirurg ist kein Forum für gesundheitspolitische Diskussionen derartiger Kalkulationen, jedoch ist die Frage der Patientensicherheit und der vermeidbaren sowie nichtvermeidbaren Operationsfolgen und Komplikationen seit langem ein Kernthema der Chirurgie. Auch in Der Chirurg wurden in den vergangenen Jahren zu verschiedenen Aspekten dieses Themas mehrere Arbeiten, u. a. in der Rubrik „Chirurgie und Recht", publiziert [4-14].

Der Herausgeber und die Schriftleitung von Der Chirurg möchten mit dem für das Jahr 2015 gewählten jahresübergreifenden Thema „Komplikationen in der Chirurgie" einen sachlichen Beitrag zu dem Komplex der operativen Sicherheit aus Sicht der Vermeidung und des Managements typischer operativer Komplikationen und ihrer juristischen Bewertung leisten. Wir möchten dabei ausdrücklich auch zur Einreichung themenbezogener Beiträge außerhalb der in den Schwerpunktthemen angesprochenen Spezialbereiche ermuntern.

Vorwärts geht es wahrlich in unserer deutschen Chirurgie; doch wenn wir keine Rückschritte machen wollen, müssen wir sehr bedächtig den Weg auf seine Sicherheit nach allen Richtungen prüfen. (Theodor Billroth am 09.08.1977 an Prof. Baum, Göttingen; [2], S. 214-217)
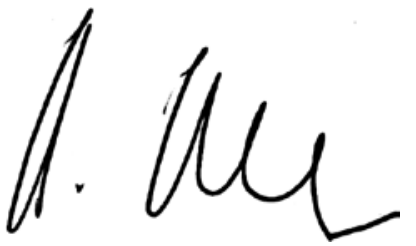

Prof. Dr. Dr. h.c. Henning Dralle

\section{Korrespondenzadresse}

Prof. Dr. Dr. h.c. H. Dralle

Universitätsklinik für Allgemein-, Viszeral- und Gefäßchirurgie, Universitätsklinikum Halle, Medizinische Fakultät, Ernst-Grube-Str. 40, 06097 Halle (Saale) henning.dralle@uk-halle.de

Interessenkonflikt. H. Dralle gibt an, dass kein Interessenkonflikt besteht.

\section{Literatur}

1. Jähne J (2014) Chirurgie zwischen Faszination, Mut und Demut. Chirurg 85:176-177

2. Fischer G (Hrsg) (1897) Briefe von Theodor Billroth. 4. Aufl. Hahnsche Buchhandlung, Hannover Leipzig

3. Leclerque G, Klauber J (2014) Einführung. In: Klauber J, Geraedts M, Friedrich J, Wasem J (Hrsg) Krankenhausreport 2014. Schattauer, S XV-XXIV

4. Theuer D, Dillschneider J, Mieth M, Büchler MW (2012) Über das geltende Haftungsrecht in der Chirurgie. Chirurg 83:54-64

5. Dillschneider J, Theuer D, Mieth M, Büchler MW (2012) Zum neuen Patientenrechtegesetz. Chirurg 83:661-666

6. Müller S, Dralle H (2013) Befunderhebungsfehler und Patientenrechtegesetz. Chirurg 84:143-148

7. Haier J, Bergmann KO (2013) Medizinrechtliche Aspekte von Tumorboards. Chirurg 84:225-230

8. Dillschneider J, Theuer D, Mieth M, Büchler MW (2013) Patientenrechtegesetz. Chirurg 84:325-326

9. Miernik A, Farin E, Kuehhas FE et al (2013) Freiburger Index für Patientenzufriedenheit. Chirurg 84:511518

10. Haier J (2013) Gegenwärtige rechtliche Rahmenbedingungen für den Betrieb und die Nutzung von Biobanken. Teil 1: Rechtsgrundlagen. Chirurg 84:785793

11. Haier J (2013) Gegenwärtige rechtliche Rahmenbedingungen für den Betrieb und die Nutzung von Biobanken. Teil 2: Datenschutz und informierte Einwilligung. Chirurg 84:892-901

12. Pieper D, Eikermann M, Mathes T et al (2014) Mindestmengen auf dem Prüfstand. Chirurg 85:121-124

13. Schneider L, Tuffs A, Büchler MW (2014) Chirurgie und Zeitgeist: Wo bleiben Ethik und Moral? Chirurg 85:224-229

14. Seidel D, Braß P, Sehnke N et al (2014) Nutzenbewertung von Medizinprodukten in der chirurgischen Praxis. Chirurg 85:407-415 